\title{
Outcomes of intraventricular hemorrhage and posthemorrhagic hydrocephalus in a population-based cohort of very preterm infants born to residents of Nova Scotia from 1993 to 2010
}

\author{
Julia A. E. Radic, MD, MPH, ${ }^{1}$ Michael Vincer, MD, ${ }^{2}$ and P. Daniel McNeely, MD'1 \\ 1Division of Neurosurgery, Department of Surgery, and 2Division of Neonatal Pediatrics, Department of Pediatrics, \\ Dalhousie University, Halifax, Nova Scotia, Canada
}

\begin{abstract}
OBJECT Intraventicular hemorrhage (IVH) is a common complication of preterm birth, and the prognosis of IVH is incompletely characterized. The objective of this study was to describe the outcomes of IVH in a population-based cohort with minimal selection bias.
\end{abstract}

METHODS All very preterm ( $\leq 30$ completed weeks) patients born in the province of Nova Scotia were included in a comprehensive database. This database was screened for infants born to residents of Nova Scotia from January 1 , 1993, to December 31, 2010. Among very preterm infants successfully resuscitated at birth, the numbers of infants who died, were disabled, developed cerebral palsy, developed hydrocephalus, were blind, were deaf, or had cognitive/ language scores assessed were analyzed by IVH grade. The relative risk of each outcome was calculated (relative to the risk for infants without IVH).

RESULTS Grades 2, 3, and 4 IVH were significantly associated with an increased overall mortality, primarily in the neonatal period, and the risk increased with increasing grade of IVH. Grade $4 \mathrm{IVH}$ was significantly associated with an increased risk of disability (RR 2.00, $p<0.001$ ), and the disability appeared to be primarily due to cerebral palsy (RR 6.07, $p<0.001$ ) and cognitive impairment (difference in mean MDI scores between Grade 4 IVH and no IVH: $-19.7, p<0.001$ ). No infants with Grade 1 or 2 IVH developed hydrocephalus, and hydrocephalus and CSF shunting were not associated with poorer outcomes when controlling for IVH grade.

CONCLUSIONS Grades 1 and 2 IVH have much better outcomes than Grades 3 or 4 , including a $0 \%$ risk of hydrocephalus in the Grade 1 and 2 IVH cohort. Given the low risk of selection bias, the results of this study may be helpful in discussing prognosis with families of very preterm infants diagnosed with IVH.

http://thejns.org/doi/abs/10.3171/2014.11.PEDS14364

KEY WORDS hydrocephalus; prematurity; intraventricular hemorrhage; outcome; vascular disorders

$\mathrm{V}$ ERY preterm infants (infants born at $\leq 30$ completed weeks' gestational age) are at increased risk of developing intraventricular hemorrhage (IVH). The current literature suggests that approximately 15\%-20\% of very preterm infants develop IVH, ${ }^{6}$ as graded by the Papile grading system based on screening ultrasonography or MRI findings..$^{8,16,25}$

Infants with IVH are known to be at increased risk of a broad range of sequelae. A significant subset will develop progressive, symptomatic posthemorrhagic hydrocephalus (PHH) requiring CSF diversion. ${ }^{20}$ These infants are also known to be at increased risk for cognitive and behavioral difficulties, including attention deficit disorders, ${ }^{10}$ and are also at increased risk of cerebral palsy (CP), lifelong disability, ${ }^{21}$ visual impairment, and seizures. ${ }^{14}$

The exact risk of developing these conditions, broken down by IVH grade, varies widely depending on the study, and a significant limitation of the literature on this sub-

ABBREVIATIONS BSID II = Bayley Scales of Infant Development, Second Edition; BSTID III = Bayley Scales of Infant and Toddler Development, Third Edition; CP = cerebral palsy; $\mathrm{CPR}$ = cardiopulmonary resuscitation; IVH = intraventricular hemorrhage; MDI = Mental Development Index (of BSID II); PFUP = Perinatal Follow-Up Program; $\mathrm{PHH}=$ posthemorrhagic hydrocephalus; $\mathrm{PVL}=$ periventricular leukomalacia.

SUBMITTED July 13, 2014. ACCEPTED November 24, 2014.

INCLUDE WHEN CITING Published online March 13, 2015; DOI: 10.3171/2014.11.PEDS14364.

DISCLOSURE The authors report no conflict of interest concerning the materials or methods used in this study or the findings specified in this paper. 
ject is that most studies are not population based..$^{20}$ Rather, they are either retrospective reviews at specific institutions or dependent upon data from the National Institutes of Health's National Institute of Child Health and Human Development Neonatal Research Network to provide a multicenter snapshot based on the experience of select academic centers. Therefore, there could be significant selection bias impacting the validity of these epidemiology reports.

The objective of this study was to accurately describe the prognosis of IVH of prematurity, by studying the population of very preterm ( $\geq 20$ and $\leq 30$ completed weeks' gestational age) infants born to residents of Nova Scotia from January 1, 1993, to December 31, 2010, who were enrolled in the IWK Health Centre Perinatal Follow-Up Program (PFUP) and whose data were included in a database with minimal selection bias and loss to follow-up.

\section{Methods}

All very preterm ( $\geq 20$ and $\leq 30$ completed weeks' gestational age) infants born to residents of Nova Scotia from January 1, 1993, onward were included in a comprehensive database. The IWK Health Centre PFUP database was screened for all very preterm infants born to residents of Nova Scotia from January 1, 1993, to December 31, 2010. Inclusion criteria included the following: gestational age of $\leq 30$ weeks but $\geq 20$ weeks and successful resuscitation at birth. Exclusion criteria included: missing information about IVH diagnosis (which only occurred when an infant died before the initial 3-day postnatal screening ultrasonography and also no autopsy was performed).

Dr. Michael Vincer, who has been in charge of this database and the perinatal follow-up program for the duration of the study period, regularly visits the only 2 other regional neonatal units that would be managing preterm infants of Nova Scotian mothers (in Moncton, New Brunswick, and Sydney, Nova Scotia) to collect data on the preterm infants treated there. Any rare preterm infant managed outside of Moncton, Sydney, or Halifax (for example, one born to a Nova Scotian mother while she was traveling elsewhere on vacation) would be routinely referred to the PFUP at the IWK hospital in Halifax once the infant and mother returned to Nova Scotia, by their pediatrician and/or family doctor.

The standard practice for the PFUP includes having a neurodevelopmental assessment complete by a neurodevelopmental team when children are 2 to 3 years of age (corrected for gestational age), including administration of the Bayley Scales of Infant Development, Second Edition (BSID II) Mental Development Index (MDI) ${ }^{3 / B a y-}$ ley Scales of Infant and Toddler Development III (BSITD III), ${ }^{2}$ with mean scores of 100 and a standard deviation of 15. The Bayley Scales were administered by certified developmental psychologists or in some cases by health care professionals with extensive experience in developmental pediatrics. The assessors were not blinded to the neonatal course or prior clinical history.

The imaging screening protocol has remained largely unchanged since 1993 in Nova Scotia. Very preterm infants are universally screened with cranial ultrasonogra- phy within 3 days of birth, at 2 weeks, at 6 weeks, and again at term. The method of ultrasound screening did change slightly starting in 2010 to include examining the brain through multiple approaches in addition to through the anterior fontanel. Furthermore, since 2010, some infants have been examined with MRI of the brain instead of an ultrasound of the head at term.

The resuscitation protocol has undergone some changes over time. A formalized neonatal resuscitation program was first initiated approximately 20 years ago. In 1999, Nova Scotia began a practice of wrapping babies in polyethylene occlusive wrap, ${ }^{23}$ and this became standard practice in 2000. In the past 10 years, there has been a reduction in oxygen use in the delivery room. Also, 15 years ago, respiratory therapists were added to the resuscitation team.

Regarding the philosophy of care for infants with IVH during the study period, the general approach in Nova Scotia has consistently been to counsel the parents of a preterm infant about prognosis and then involve them in decisions regarding goals of care for their child. This general approach has not significantly changed over the study period.

Of note, over time in Nova Scotia there has been a significant increase in antenatal corticosteroid usage, birth weight, use of cardiopulmonary resuscitation (CPR), dobutamine usage, dopamine usage, infant respiratory distress syndrome, maternal weight, maternal height, surfactant usage, high-flow oscillatory ventilation usage, birth asphyxia, necrotizing enterocolitis, twin pregnancies, nitric oxide use in the infant, paralyzing agent usage, and phototherapy usage. Over the study period, there was also decreasing intrapartum magnesium sulfate usage, maternal fever during labor, and suspected intrauterine growth restriction on ultrasound.

The numbers of infants who were successfully resuscitated at birth, who were diagnosed with IVH, who died, who were lost to follow-up, and who were disabled at the final 2- to 3-year neurodevelopmental assessment were extracted from the database (Fig. 1). Disability was defined as having CP, psychology scores (BSID II MDI scores or BSITD III cognitive/language scores) $\geq 1$ SD below the mean, bilateral deafness, or bilateral blindness.

The primary outcome measures were: 1) overall mortality during the follow-up period and 2) any disability at final 2- to 3-year follow-up assessment. For descriptive and prognostic purposes, mortality and disability were subdivided.

Mortality was subdivided into: a) neonatal death (death from birth to 28 days), b) infant death (death from 29 to 365 days), and c) death from 366 days to final follow-up. Disability was subdivided into a) mild disability (mild CP, and/or psychology scores 1 to $<2$ SD below the mean), b) moderate disability (moderate CP [can walk but with difficulty] and/or psychology scores 2-3 SD below the mean, and/or bilateral deafness), and c) severe disability (severe $\mathrm{CP}$ [severely impaired ambulation or nonambulatory] and/ or psychology scores $>3$ SD below the mean and/or bilateral blindness).

Secondary outcome measures were: 1) CP, with moderate/severe $\mathrm{CP}$ as a subdivision, 2) hydrocephalus (defined 


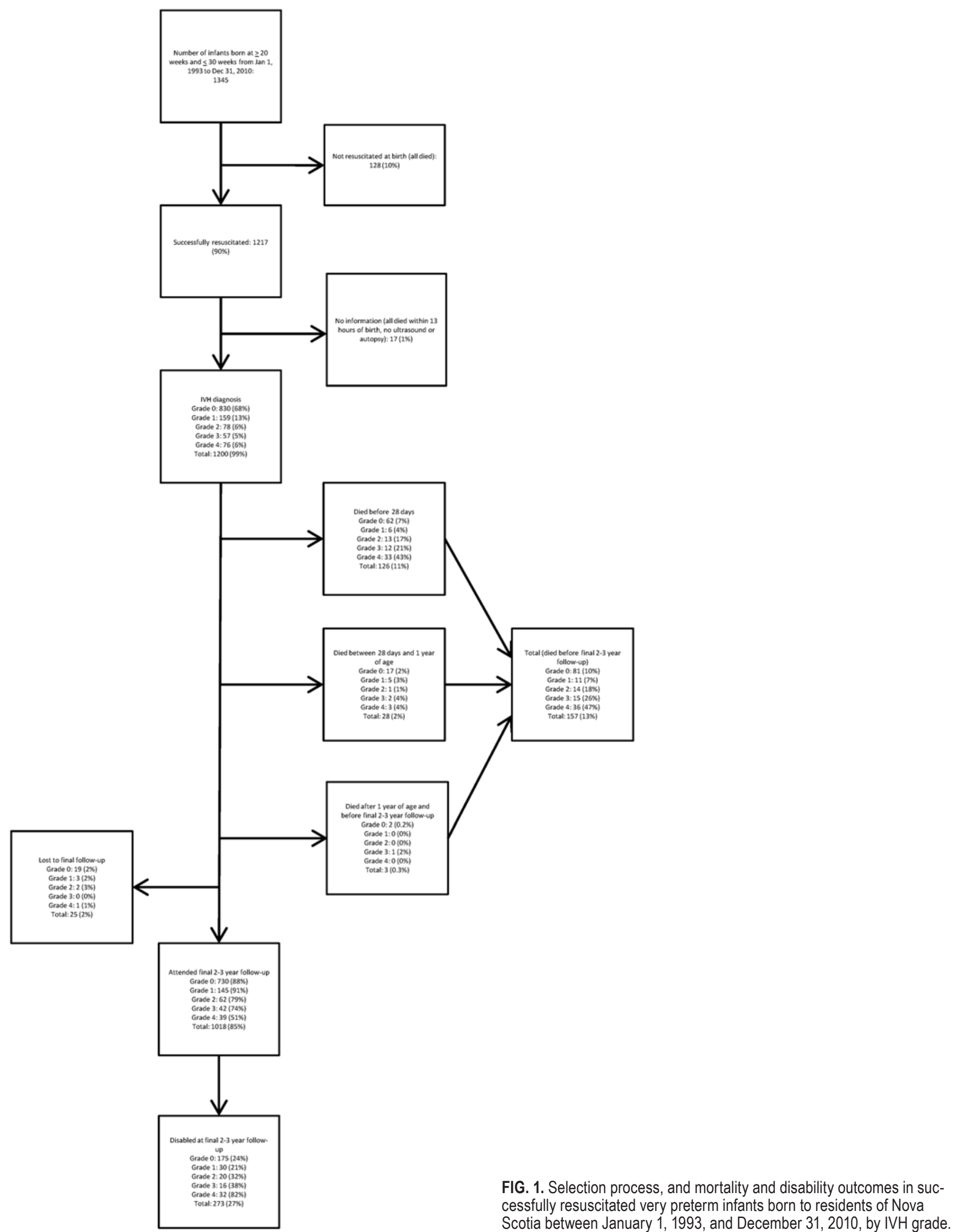


as ventricle size above the 97th percentile for gestational age) and clinical signs of raised intracranial pressure, , 5,13,20 with shunt-treated hydrocephalus as a subdivision, 3) blindness, 4) deafness, and 5) MDI score corrected for gestational age, obtained at final 2- to 3-year follow-up. MDI scores were obtained directly from BSID II scores and indirectly from BSITD III scores, by converting the BSITD III cognitive and language composite scores to an MDI-equivalent score using the following formula ${ }^{15}$ : MDI $=88.8-\left(61.6^{*}[\text { language score } / 100]^{-1}\right)+(0.67 *$ cognitive score).

The number of infants with each of these outcomes at final follow-up was extracted from the database, and the risk of developing each outcome was calculated and broken down by IVH grade. Relative risks of developing each binary outcome measure with IVH versus without IVH were calculated and subdivided by IVH grade. These relative risks were analyzed for statistical significance using the chi-square (chi-square) test or, if the chi-square assumptions did not hold, the Fisher's exact test for categorical outcome variables. For outcome variables with missing data, relative risk and $p$ values were calculated without including the missing data. Sensitivity analyses were also carried out to assess whether a significant association between IVH grade and the outcome would occur if the infants with missing data either did not incur the outcome or did incur the outcome, and if the infants with missing data with IVH did incur the outcome while the infants without IVH did not, and if the infants with missing data with IVH did not incur the outcome while the infants without IVH did. The chi-square test for trend was used to assess for evidence of a trend in association between overall mortality, disability, CP, hydrocephalus, blindness, and deafness, and increasing IVH grade. The chi-square test for trend was also used to assess for changes in overall mortality, withdrawal rates, and overall disability over time, in all very preterm infants and also in infants with Grade 4 of IVH.

For continuous outcome variables, the mean and standard deviation of the outcome were calculated for each IVH grade. Differences in mean values were calculated for infants with each grade of IVH compared with infants without IVH. Statistical significance of these differences was assessed using a 2-tailed Student t-test or Wilcoxon rank-sum test depending on data normality.

Multivariate logistic regression, controlling for IVH grade, was used on the subgroup of infants with Grade 3 or $4 \mathrm{IVH}$ who were not lost to follow-up, to assess whether hydrocephalus or shunt surgery were associated with an increased risk of negative outcome in this subgroup. Multivariate logistic regression, controlling for IVH grade, was also used on the subgroup of infants with Grade 3 or $4 \mathrm{IVH}$ with hydrocephalus who were not lost to follow-up, to assess whether shunt surgery was associated with an increased risk of negative outcome in this subgroup.

Univariate logistic regression was used to identify the associations between several infant, maternal, obstetrical, and management variables and overall disability, overall mortality, and IVH. Multivariate logistic regression was used to study the association between IVH and disability, controlling for all the variables significantly associated with both IVH and disability. Multivariate logistic regression was also used to study the association between IVH and overall mortality, controlling for all the variables significantly associated with both IVH and mortality.

All data analysis were carried out using STATA 11.2 (StataCorp LP), and the threshold for statistical significance was set at $\alpha=0.05$. Ethics approval for this research was obtained from the IWK Health Centre Research Ethics Board.

\section{Results}

Figure 1 illustrates the selection process for this study. Cases involving all very preterm infants born from 1993 onward were entered in the PFUP database and screened for inclusion in this study. Ultimately, 1200 cases fulfilled the criteria for inclusion and were included in the analysis. Only 25 infants (2\%) were lost to follow-up.

The mortality and disability outcomes for all infants included in the study by IVH grade are pictured in Fig. 2 . The number of infants experiencing each primary and secondary outcome measure as well as relative risks of developing the outcome by IVH grade (relative to the risk for infants without IVH) are detailed in Table 1.

The overall risk of mortality was similar in infants with no IVH (10\%) and with Grade 1 IVH (7\%), and it was significantly increased in infants with Grade $2(18 \%)$, Grade $3(26 \%)$, and Grade $4(47 \%)$ IVH. The overall risk of disability was similar in infants with no IVH (21\%), Grade 1 (19\%), Grade 2 (26\%), and Grade 3 IVH (28\%), but it was significantly increased in infants with Grade 4 IVH (42\%). The risk of $\mathrm{CP}$ was significantly increased in infants with Grade 2 IVH (13\%), Grade 3 IVH (18\%), and Grade 4 IVH (39\%) compared with infants without IVH (7\%), although the risk of moderate or severe $\mathrm{CP}$ was only increased in infants with Grade 4 IVH (12\% vs $2 \%$ of infants without IVH). Only infants with Grade 3 or 4 IVH developed hydrocephalus (21\% and 37\%, respectively), and only $12 \%$ and $25 \%$ of infants with Grade 3 and Grade 4 IVH were treated with CSF shunting. Only Grade 4 IVH was associated with a significantly lower mean MDI score compared with the mean score in infants without IVH $(77.5 \pm 23.4$ vs $97.2 \pm 18.8)$.

The chi-square test for trend was significant for increasing odds of mortality $(\mathrm{p}<0.001)$, disability $(\mathrm{p}<0.001)$, CP $(\mathrm{p}<0.001)$, and hydrocephalus $(\mathrm{p}<0.001)$, associated with increasing IVH grade. If the infants with missing data were left out of the analysis, there was no significant trend found for blindness $(p=0.159)$ or deafness $(p=0.080)$, and IVH (of any grade) was not significantly associated with either blindness or deafness. However, on sensitivity analyses looking at the missing data, significant associations between each grade of IVH and blindness or deafness could be elicited, throwing into question the validity of the associations between IVH and blindness/deafness.

Overall mortality in very preterm infants has significantly decreased over time, from $17.4 \%$ (65 of 374 infants) during 1993-1997 to $7.7 \%$ (16 of 208) during 2008-2010 $(\mathrm{p}<0.001)$. Overall mortality in very preterm infants with Grade 4 IVH (risk 47\%) has not changed significantly over time $(\mathrm{p}=0.152)$. 


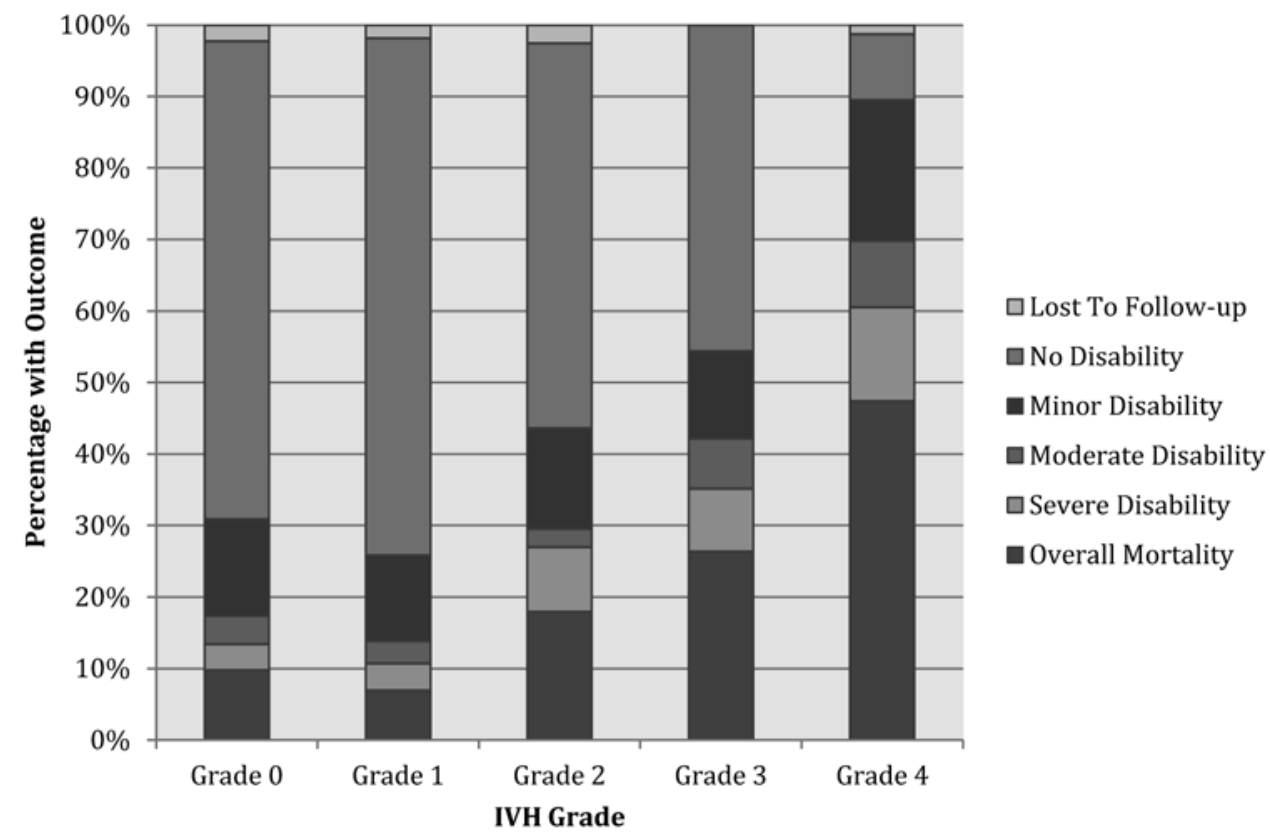

FIG. 2. Primary outcomes of IVH, by grade of hemorrhage. Outcomes are broken down into percentage of very preterm infants of each grade of IVH who died, were disabled, or were lost to follow up by final 2- to 3-year follow-up. Note "Grade 0" IVH means no $\mathrm{IVH}$.

The rate of withdrawal of care in IVH is $15.1 \%$ for all infants with IVH, and $73.7 \%$ for the infants with IVH who died. These 2 rates have not significantly changed over time $(\mathrm{p}=0.287$ and $\mathrm{p}=0.073$ respectively). The rate of withdrawal of care in IVH Grade 4 is $39.47 \%$ for all infants with Grade $4 \mathrm{IVH}$, and $83.3 \%$ for infants with Grade $4 \mathrm{IVH}$ who died. These 2 rates have not significantly changed over time ( $p=0.475$ and $p=0.275$, respectively).

The overall rate of subsequent disability for very preterm infants has significantly increased over time from $13.9 \%$ (52 of 374 infants) for 1993-1997 to $36.1 \%$ (75 of 208) for 2008-2010 ( $<<0.001)$. The rate of subsequent disability in very preterm infants with Grade $4 \mathrm{IVH}$ (risk $42 \%)$ has not significantly changed over time $(\mathrm{p}=0.328)$.

On multivariate logistic regression, in the subgroup of infants with Grade 3 or $4 \mathrm{IVH}$, controlling for IVH grade, hydrocephalus was associated with reduced risk of overall mortality (OR $0.21, \mathrm{p}=0.001$ ) but was not significantly associated with any of the morbidity outcome measures. Shunt surgery was also associated with reduced risk of overall mortality (OR $0.12, \mathrm{p}=0.001$ ) but was not significantly associated with any of the morbidity outcome measures.

On multivariate logistic regression, in the subgroup of infants with Grade 3 or 4 IVH with hydrocephalus, controlling for IVH grade, shunt surgery was not significantly associated with any of the outcome measures.

Table 2 illustrates the associations between various infant, maternal, obstetrical, and management variables and overall disability, overall mortality, and IVH. On multivariate logistic regression looking at the association between IVH and overall mortality and controlling for variables significantly associated with both IVH and death, only CPR (OR 11.3, $\mathrm{p}=0.005)$, high-flow oscillatory ventilation usage (OR $1.00, \mathrm{p}=0.022)$, and bronchopulmonary dysplasia (OR $0.15, \mathrm{p}=0.049$ ) remained significantly associated with overall mortality. IVH dropped out of the model and was no longer significantly associated with mortality.

On multivariate logistic regression looking at the association between IVH and overall disability and controlling for variables significantly associated with both IVH and disability, only male sex (OR 1.68, $\mathrm{p}=0.011)$, surfactant usage (OR 1.76, $\mathrm{p}=0.043$ ), and cystic periventricular leukomalacia (PVL) (OR 27.0, $\mathrm{p}<0.001)$ remained significantly associated with overall disability. IVH, hydrocephalus, and shunt surgery all fell out of the model and were no longer significantly associated with disability.

\section{Discussion}

We found IVH to be associated with an increased risk of death, disability, CP, hydrocephalus, and a lower MDI score, and the risk increases with increasing grade of IVH. This is consistent with previously published studies examining neurodevelopmental outcomes of IVH. ${ }^{4,9,18,20}$ However, unique to our study, only Grade 4 IVH was associated with an increased risk of overall disability, moderate/ severe $\mathrm{CP}$, and a worse MDI score compared with infants without IVH, and there was no association between IVH diagnosis and blindness or deafness. Infants with lower grades of IVH (Grades 1, 2, and 3) had similar neurodevelopmental outcomes to infants without IVH, except for an increased risk of mild CP in infants with Grades 2 and 3 IVH.

In a recently published study, Payne et al. ${ }^{18}$ looked at an American multicenter experience and also found no association with low grade IVH (Grades 1 and 2) and worse neurodevelopmental outcomes at 18-24 months of age for 


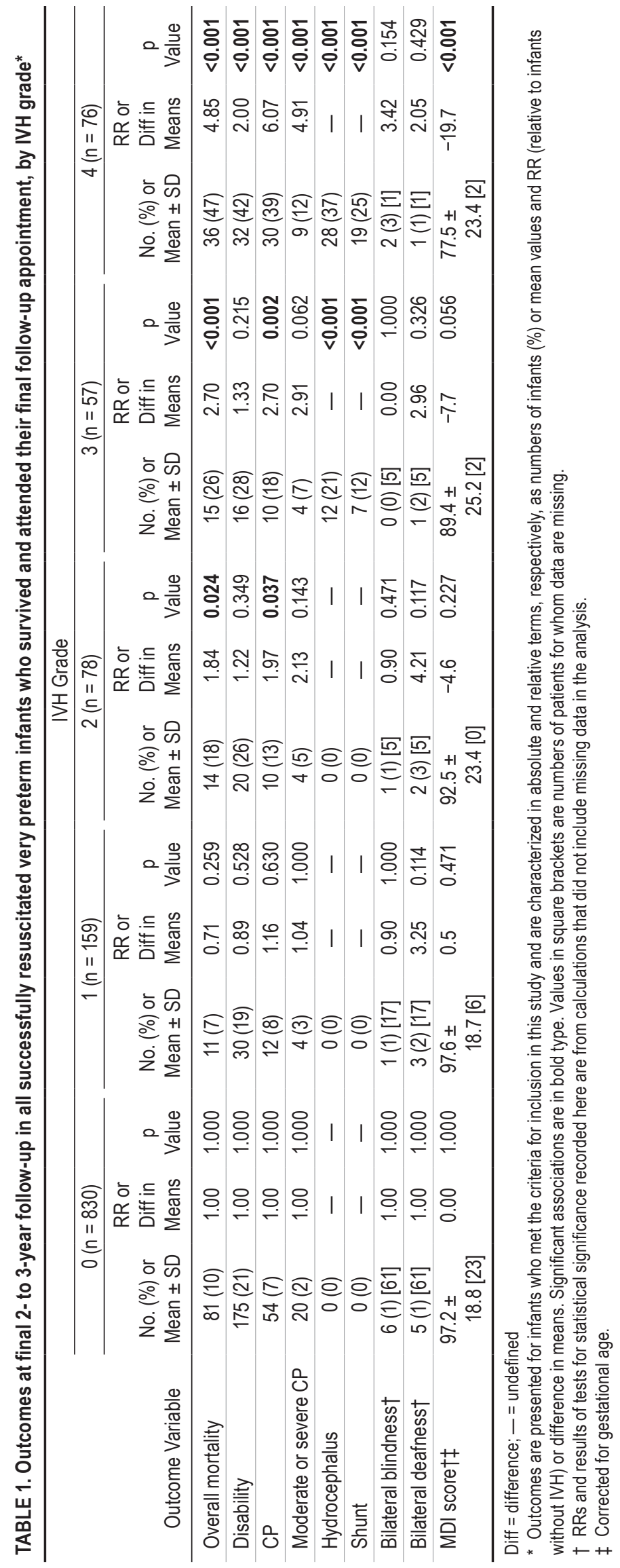


TABLE 2. Associations between various infant, maternal, obstetrical, and management variables and overall disability, overall mortality, and IVH diagnosis*

\begin{tabular}{|c|c|c|c|c|c|c|}
\hline Variable & $\begin{array}{c}\text { OR for } \\
\text { Disability }\end{array}$ & $\begin{array}{c}\text { p Value for Assoc } \\
\text { btwn Disability \& } \\
\text { Variable }\end{array}$ & $\begin{array}{l}\text { OR for } \\
\text { Death }\end{array}$ & $\begin{array}{l}\text { p Value for Assoc } \\
\text { btwn Death \& } \\
\text { Variable }\end{array}$ & $\begin{array}{l}\text { OR for } \\
\text { IVH }\end{array}$ & $\begin{array}{c}\text { p Value of Assoc } \\
\text { btwn IVH \& } \\
\text { Variable }\end{array}$ \\
\hline Time (5-yr epochs) & 1.40 & $<0.001$ & 0.74 & $<0.001$ & 1.16 & 0.011 \\
\hline Antenatal corticosteroid use & 0.52 & 0.001 & 0.31 & 0.063 & 0.62 & 0.006 \\
\hline Birth weight $(\mathrm{g})$ & 0.9995 & 0.034 & 0.9960 & $<0.001$ & 0.9993 & $<0.001$ \\
\hline Any CPR & 4.54 & $<0.001$ & 6.76 & $<0.001$ & 2.75 & $<0.001$ \\
\hline Dobutamine use & 6.58 & $<0.001$ & 9.69 & $<0.001$ & 3.23 & $<0.001$ \\
\hline Dopamine use & 3.81 & $<0.001$ & 8.36 & $<0.001$ & 2.62 & $<0.001$ \\
\hline Gestational age (wks) & 0.87 & $<0.001$ & 0.56 & $<0.001$ & 0.82 & $<0.001$ \\
\hline Infant respiratory distress syndrome & 2.43 & $<0.001$ & 1.61 & 0.106 & 1.34 & 0.133 \\
\hline Cesarean section & 1.12 & 0.416 & 0.53 & $<0.001$ & 0.41 & $<0.001$ \\
\hline Maternal age (yrs) & 0.98 & 0.099 & 0.96 & 0.011 & 0.976 & 0.025 \\
\hline Maternal weight (kg) & 1.00 & 0.447 & 1.00 & 0.904 & 0.9984 & 0.702 \\
\hline Maternal height (m) & 0.63 & 0.662 & 1.17 & 0.934 & 0.65 & 0.658 \\
\hline Male sex & 1.79 & $<0.001$ & 1.07 & 0.686 & 1.34 & 0.020 \\
\hline Surfactant use & 2.41 & $<0.001$ & 2.70 & $<0.001$ & 1.52 & 0.003 \\
\hline High-flow oscillatory ventilation & 1.00 & $<0.001$ & 1.0046 & $<0.001$ & 1.003 & 0.002 \\
\hline Gestational hypertension & 0.78 & 0.178 & 0.27 & $<0.001$ & 0.62 & 0.006 \\
\hline Maternal fever during labor (temp $>38^{\circ} \mathrm{C}$ ) & 0.66 & 0.254 & 1.32 & 0.433 & 1.98 & 0.010 \\
\hline Suspected IUGR on US & 1.55 & 0.215 & 2.11 & 0.036 & 0.45 & 0.041 \\
\hline Birth asphyxia & 2.06 & $<0.001$ & 5.39 & $<0.001$ & 1.58 & 0.002 \\
\hline Necrotizing enterocolitis & 2.07 & 0.061 & 2.13 & 0.054 & 2.31 & 0.011 \\
\hline Intrapartum $\mathrm{MgSO}_{4}$ use & 0.66 & 0.032 & 0.68 & 0.126 & 0.48 & $<0.001$ \\
\hline Twin pregnancy & 1.11 & 0.489 & 1.22 & 0.283 & 0.90 & 0.443 \\
\hline Nitric oxide use in baby & 3.64 & $<0.001$ & 8.41 & $<0.001$ & 2.44 & $<0.001$ \\
\hline Paralyzing agent & 4.69 & $<0.001$ & 3.98 & $<0.001$ & 2.53 & $<0.001$ \\
\hline Phototherapy & 1.64 & 0.066 & 6.81 & $<0.001$ & 0.99667 & 0.987 \\
\hline BPD at 28 days & 1.72 & 0.002 & 4.29 & 0.006 & 1.80 & 0.001 \\
\hline Septicemia in baby & 1.20 & 0.208 & 0.65 & 0.029 & 1.23 & 0.117 \\
\hline Hydrocephalus & 7.00 & $<0.001$ & 1.64 & 0.219 & - & - \\
\hline Shunt surgery & 8.59 & $<0.001$ & 0.86 & 0.813 & - & - \\
\hline Cystic PVL & 18.4 & $<0.001$ & 0.95 & 0.866 & 4.22 & $<0.001$ \\
\hline
\end{tabular}

Assoc = association; BPD = bronchopulmonary dysplasia; IUGR = intrauterine growth restriction; temp = temperature; US = ultrasound.

* Odds ratios $(\mathrm{OR})$ and $p$ values were calculated using univariate logistic regression analysis. Significant $p$ values are in boldface.

infants born between 2006 and 2008. However, this study was not population based and so may have contained referral bias, and it also had a higher rate of loss to follow-up $(13 \%)$. A second significant recently published study, by Bolisetty et al., ${ }^{4}$ was a regional cohort study of all infants admitted to all 10 tertiary neonatal intensive care units in New South Wales and the Australian Capital Territory between 1998 and 2004, and unlike our study, it showed an increased risk of worse neurodevelopmental outcomes for infants with low-grade IVH. However, the study had significant loss to follow-up (25\%), may have missed infants not admitted to a tertiary neonatal intensive care unit, and excluded infants with congenital malformations. Perhaps infants with better outcomes were lost to follow-up, falsely making it appear as if low-grade IVH is associated with worse outcomes.
Other centers with potential referral bias and greater rates of loss to follow-up have also found worse neurodevelopmental outcomes with low-grade IVH, ${ }^{17}$ while some centers have found worse outcomes in terms of disability only with the higher grades of IVH and with white matter changes such as cystic PVL, similar to our findings.?

In addition, though most published studies show an association between hydrocephalus/shunt surgery and worse neurodevelopmental outcomes, $, 11,20$ in our study, in infants with IVH Grade 3 or 4 , hydrocephalus and shunt surgery were not independently associated with an increased risk of morbidity. Hydrocephalus and shunt surgery were, however, independently associated with a reduced risk of overall mortality, though this is likely due to the fact that surviving infants are more likely to progress to develop clinical hydrocephalus and require a shunt. 
Not surprisingly, we found that mortality of very preterm infants in Nova Scotia has been decreasing over time while disability has been increasing. This is consistent with the experience observed at other centers. ${ }^{27}$ This trend does not seem to be explained by a change in rates of withdrawal of care in Nova Scotia in infants with IVH, as the risk of withdrawal of care has not significantly changed over time. There have been several other factors however that have changed over time, including variables such as increased use of cardiopulmonary resuscitation, increased use of paralytics and inotropes, and increased risk of birth asphyxia. These may be also contributing to decreasing mortality at the expense of increasing disability.

Interestingly, in the multivariate regression analysis examining factors that may contribute to disability, cystic PVL is by far the strongest predictor of disability, with an odds ratio of 27.0 for disability, to the point of pushing IVH, PHH, and CSF shunting into statistical insignificance. This is also consistent with the experience at other centers ${ }^{7,19,26}$ and fits with the developing understanding that PVL (with or without IVH) is potentially the most significant driver of disability in very preterm surviving infants. ${ }^{1,19,24}$ Nonetheless, PVL is diagnosed later than IVH at 3-4 weeks of age, ${ }^{9}$ and therefore this study was instead focused primarily on the prognosis of IVH, independent of future PVL status, to facilitate discussion of prognosis with families of infants with this diagnosis.

The strengths of this study are the low loss to follow-up rate $(2 \%)$ and the fact that every very preterm infant born to a resident of Nova Scotia is included in this database, making it a strong population-based study. Because of this, there is a very low risk of selection bias, and hence this is a valid representation of the 2- to 3-year long-term prognosis of IVH of prematurity in Nova Scotia. The results should therefore be generalizable to other regions of the world with similar management protocols for preterm infants and similar population demographics.

This study is limited by the lack of information on the timing of IVH diagnoses, mortality, and the various outcomes, precluding the ability to do survival analysis or Cox regression. It is also therefore difficult to fully understand whether a significant association between IVH diagnosis and an outcome measures is due to the timing of the outcome before or after IVH diagnosis. The results of this analysis are also only applicable to infants born at less than 31 weeks gestational age, because this was the selection criteria for enrollment in the PFUP. However, this is still more inclusive when compared with other recent studies on this topic, which included infants born at less than 27-29 weeks gestational age, ${ }^{4,18}$ and hence is more likely reflective of the overall outcomes of all infants with IVH of prematurity.

Another limitation of our study was that there was also significant missing outcome data on deafness and blindness in particular $(0 \%-10 \%$ per IVH grade), making the associations between IVH grade and deafness/blindness more prone to bias. This may also explain why we did not find an association between IVH and blindness/deafness, unlike other groups. ${ }^{4} \mathrm{~A}$ substantial quantity of data was also missing for several variables, such as maternal smoking/drinking/substance abuse, socioeconomic status, and race. These factors could have confounded the associations between IVH and the various outcome measures, ${ }^{22}$ though a recent Canadian study suggested socioeconomic status may not affect mortality/disability outcomes in preterm infants in Canada. ${ }^{12}$ Nonetheless, it might have been helpful to control for these variables in the multivariate analyses.

\section{Conclusions}

In conclusion, IVH is associated with increased disability and mortality, and the risk is higher with higher grades of hemorrhage. Given the strong population-based nature of this study, the risks of the various outcomes by IVH grade described in this paper can be used to discuss prognosis with families of very preterm infants with IVH in similar settings to Nova Scotia.

\section{References}

1. Bass WT, Jones MA, White LE, Montgomery TR, Aiello F III, Karlowicz MG: Ultrasonographic differential diagnosis and neurodevelopmental outcome of cerebral white matter lesions in premature infants. J Perinatol 19:330-336, 1999

2. Bayley N: Bayley Scales of Infant and Toddler Development, ed 3. San Antonio, TX: Harcourt Assessment, 2006

3. Bayley N: Bayley Scales of Infant Development, ed 2. San Antonio, TX: Psychological Corp, 1993

4. Bolisetty S, Dhawan A, Abdel-Latif M, Bajuk B, Stack J, Lui K: Intraventricular hemorrhage and neurodevelopmental outcomes in extreme preterm infants. Pediatrics 133:55-62, 2014

5. de Vries LS, Liem KD, van Dijk K, Smit BJ, Sie L, Rademaker KJ, et al: Early versus late treatment of posthaemorrhagic ventricular dilatation: results of a retrospective study from five neonatal intensive care units in The Netherlands. Acta Paediatr 91:212-217, 2002

6. du Plessis AJ: The role of systemic hemodynamic disturbances in prematurity-related brain injury. J Child Neurol 24:1127-1140, 2009

7. Dyet LE, Kennea N, Counsell SJ, Maalouf EF, Ajayi-Obe M, Duggan PJ, et al: Natural history of brain lesions in extremely preterm infants studied with serial magnetic resonance imaging from birth and neurodevelopmental assessment. Pediatrics 118:536-548, 2006

8. El-Dib M, Massaro AN, Bulas D, Aly H: Neuroimaging and neurodevelopmental outcome of premature infants. Am J Perinatol 27:803-818, 2010

9. Enzmann D, Murphy-Irwin K, Stevenson D, Ariagno R, Barton J, Sunshine P: The natural history of subependymal germinal matrix hemorrhage. Am J Perinatol 2:123-133, 1985

10. Indredavik MS, Vik T, Evensen KA, Skranes J, Taraldsen G, Brubakk AM: Perinatal risk and psychiatric outcome in adolescents born preterm with very low birth weight or term small for gestational age. J Dev Behav Pediatr 31:286-294, 2010

11. Jary S, De Carli A, Ramenghi LA, Whitelaw A: Impaired brain growth and neurodevelopment in preterm infants with posthaemorrhagic ventricular dilatation. Acta Paediatr 101:743-748, 2012

12. Ko G, Shah P, Kovacs L, Ojah C, Riley P, Lee SK: Neighbourhood income level and outcomes of extremely preterm neonates: protection conferred by a universal health care system. Can J Public Health 103:e443-e447, 2012

13. Levene MI: Measurement of the growth of the lateral ventricles in preterm infants with real-time ultrasound. Arch Dis Child 56:900-904, 1981 
14. Maitre NL, Marshall DD, Price WA, Slaughter JC, O'Shea TM, Maxfield C, et al: Neurodevelopmental outcome of infants with unilateral or bilateral periventricular hemorrhagic infarction. Pediatrics 124:e1153-e1160, 2009

15. Moore T, Johnson S, Haider S, Hennessy E, Marlow N: Relationship between test scores using the second and third editions of the Bayley Scales in extremely preterm children. J Pediatr 160:553-558, 2012

16. Papile LA, Burstein J, Burstein R, Koffler H: Incidence and evolution of subependymal and intraventricular hemorrhage: a study of infants with birth weights less than 1,500 gm. J Pediatr 92:529-534, 1978

17. Patra K, Wilson-Costello D, Taylor HG, Mercuri-Minich N, Hack M: Grades I-II intraventricular hemorrhage in extremely low birth weight infants: effects on neurodevelopment. J Pediatr 149:169-173, 2006

18. Payne AH, Hintz SR, Hibbs AM, Walsh MC, Vohr BR, Bann $\mathrm{CM}$, et al: Neurodevelopmental outcomes of extremely lowgestational-age neonates with low-grade periventricular-intraventricular hemorrhage. JAMA Pediatr 167:451-459, 2013

19. Perlman JM: White matter injury in the preterm infant: an important determination of abnormal neurodevelopment outcome. Early Hum Dev 53:99-120, 1998

20. Robinson S: Neonatal posthemorrhagic hydrocephalus from prematurity: pathophysiology and current treatment concepts. J Neurosurg Pediatr 9:242-258, 2012

21. van Haastert IC, Groenendaal F, Uiterwaal CS, Termote JU, van der Heide-Jalving M, Eijsermans MJ, et al: Decreasing incidence and severity of cerebral palsy in prematurely born children. J Pediatr 159:86-91.e1, 2011

22. Vohr B, Garcia Coll C, Flanagan P, Oh W: Effects of intraventricular hemorrhage and socioeconomic status on perceptual, cognitive, and neurologic status of low birth weight infants at 5 years of age. J Pediatr 121:280-285, 1992

23. Vohra S, Frent G, Campbell V, Abbott M, Whyte R: Effect of polyethylene occlusive skin wrapping on heat loss in very low birth weight infants at delivery: a randomized trial. J Pediatr 134:547-551, 1999
24. Vollmer B, Roth S, Baudin J, Stewart AL, Neville BG, Wyatt JS: Predictors of long-term outcome in very preterm infants: gestational age versus neonatal cranial ultrasound. Pediatrics 112:1108-1114, 2003

25. Volpe JJ: Neurology of the Newborn, ed 5. Philadelphia: Saunders Elsevier, 2008

26. Whitaker AH, Feldman JF, Van Rossem R, Schonfeld IS, Pinto-Martin JA, Torre C, et al: Neonatal cranial ultrasound abnormalities in low birth weight infants: relation to cognitive outcomes at six years of age. Pediatrics 98:719-729, 1996

27. Wilson-Costello D, Friedman H, Minich N, Fanaroff AA, Hack M: Improved survival rates with increased neurodevelopmental disability for extremely low birth weight infants in the 1990s. Pediatrics 115:997-1003, 2005

\section{Author Contributions}

Conception and design: all authors. Acquisition of data: Vincer. Analysis and interpretation of data: Radic. Drafting the article: Radic. Critically revising the article: all authors. Reviewed submitted version of manuscript: all authors. Approved the final version of the manuscript on behalf of all authors: Radic. Statistical analysis: Radic. Study supervision: Vincer, McNeely.

\section{Supplemental Information}

Previous Presentation

Portions of this work were presented in poster presentation form at the Canadian Neurological Sciences Federation meeting in Banff, Alberta, on June 6, 2014.

\section{Correspondence}

Julia A. E. Radic, Division of Neurosurgery, IWK Health Centre, 5850 University Ave., Halifax, NS B3K 6R8, Canada. email: jradic@dal.ca. 